\title{
Co-existence of Hypertension and Pre-existing Cardiovascular Disease and Mortality in Patients on Continuous Ambulatory Peritoneal Dialysis
}

\section{Xiaoyang Wang}

Zhengzhou University

\section{Xiaojiang Zhan}

Nanchang University

Qing Zhou

Sun Yat-Sen University

\section{Xiaoran Feng}

Nanchang University

\section{FenFen Peng}

Southern Medical University

Niansong Wang

Shanghai Jiao Tong University

\section{Yueqiang Wen}

Guangzhou Medical University

Xianfeng Wu ( $\square$ xianfengwu2@163.com )

Shanghai Jiao Tong University

\section{Research article}

Keywords: dialysis, mortality, hypertension, cardiovascular disease

Posted Date: June 10th, 2020

DOI: https://doi.org/10.21203/rs.3.rs-34311/v1

License: (1) (1) This work is licensed under a Creative Commons Attribution 4.0 International License. Read Full License 


\section{Abstract \\ Background}

Little is known about whether co-existence of hypertension (HTN) and pre-existing cardiovascular disease (CVD) has a more harmful effect on mortality compared with either comorbidity alone in patients on continuous ambulatory peritoneal dialysis (CAPD).

\section{Methods}

We conducted a retrospective study of 3073 incident Chinese patients on CAPD from five dialysis centers between January 1, 2005 and December 31, 2018 in a real-world setting. The primary and secondary outcomes were all-cause and CVD mortality. The association between interesting comorbidities and mortality was analyzed using Cox regression models and the Fine and Gray competing risk models.

\section{Results}

Over a median of 33.7 months of follow-up, 581 (18.6\%) patients died, with 286 (9.3\%) CVD mortality. The incidence of all-cause mortality was $32.2,56.1,74.4$, and 131.0/1000 patient-years, and the incidence of CVD mortality was $15.0,28.2,34.7$, and $69.6 / 1000$ patient-years in the control group (those without either hypertension or CVD), HTN group, CVD group, and HTN plus CVD group respectively. After adjusting for the confounding factors, HTN plus CVD, CVD, and HTN groups had a higher risk of all-cause mortality (HR 3.98, 95\% $\mathrm{Cl} 3.07$ to 5.17 ; HR 2.18, 95\% $\mathrm{Cl} 1.27$ to 3.74 ; and $\mathrm{HR} 1.83,95 \% \mathrm{Cl} 1.47$ to 2.28 ) and CVD mortality (HR 4.68, 95\% Cl 3.27 to 6.69 ; HR 2.11, 95\% Cl 0.96 to 4.63 ; and HR 1.87, 95\% $\mathrm{Cl} 1.37$ to 2.54 ), respectively, compared to the control group. Similar findings were observed using the Fine and Gray competing risk models. There was no significant interaction between HTN and CVD on all-cause and CVD mortality $(\beta=0.010, P=0.973 ; \beta=0.058, P=0.892)$ in the study population.

\section{Conclusions}

Among CAPD patients, co-existence of HTN and pre-existing CVD at the start of CAPD had a more harmful effect on mortality compared to either HTN or pre-existing CVD alone, and pre-existing CVD may have also a more harmful effect on mortality than HTN.

\section{Introduction}

Although renal replacement therapy (RRT) has been significantly improved in recent decades, the overall prognosis of end stage renal disease (ESRD) remains poor, with only $11 \%$ of peritoneal dialysis patients surviving past 10 years [1]. Cardiovascular disease (CVD) accounts for approximately $50 \%$ of deaths in dialysis patients [2]. Dialysis patients have 10 to 30 times of CVD mortality than the general population, 
even after adjusting for age, gender, and ethnicity [3]. Dialysis patients have a high prevalence of traditional CVD risk factors, such as hypertension (HTN), pre-existing CVD, diabetes mellitus, etc. The presence of HTN affects approximately $70.0 \%$ of Chinese patients on continuous ambulatory peritoneal dialysis (CAPD) [4, 5]. Elevated, lower or uncontrolled blood pressure (BP) is highly prevalent among dialysis patients and is associated with increased mortality in this population [6-8]. Meanwhile, chronic kidney disease (CKD) has been identified as an independent risk factor for CVD, resulting in twice as likely to develop CVD compared to the general population [9-11]. So, patients may have a higher prevalence of pre-existing CVD, when receiving RRT. Dialysis patients with pre-existing CVD have poorer survival compared to those without pre-existing CVD $[12,13]$. Interestingly, among CAPD patients, we wonder whether co-existence of HTN and pre-existing CVD has a more harmful effect on mortality than HTN or pre-existing CVD alone, and pre-existing CVD is more strongly associated with mortality than HTN. The objective of this study was to evaluate (1) the association between the co-existence of HTN and preexisting CVD at the start of dialysis and mortality, (2) the association between pre-existing CVD and HTN and mortality in CAPD patients.

\section{Materials And Methods}

\section{Study Design and Population}

We conducted a retrospective cohort study of 3073 incident CAPD patients from five PD centers of three provinces in China, between January 1,2005, and December 31, 2018. The inclusion criteria were relatively broad, which implies that the patients may be reasonably representative for the CAPD population. On behalf of the real world setting of CAPD, no patient were excluded in this study. The study was approved by the Human Ethics Committee of each research center, consistent with the ethical principles of the Declaration of Helsinki.

\section{Data Collection and Definitions}

Data on demographics, comorbid conditions, medications, and laboratory values at the start of CAPD were abstracted from medical records by two trained investigators in each center using uniform and standardized data collection tools: demographic characteristics (age, sex, body mass index, systolic BP, diastolic BP, 24-hour urine volume, current smoking, and current alcohol consumption); comorbidities (diabetes mellitus, pre-existing CVD, HTN, hyperlipidemia); medications (calcium channel blockers, beta blockers, angiotensin II receptor blockers/angiotensin-converting enzyme inhibitors [ACEI/ARBs], diuretics, statins, and aspirin); and laboratory variables (hemoglobin, serum albumin, serum uric acid, estimated glomerular filtration rate [eGFR], cholesterol, triglyceride, high density lipoprotein, low density lipoprotein, high-sensitivity C-reactive protein [hs-CRP]). The diagnosis of HTN was defined as pre-dialysis systolic BP measurements $>150 \mathrm{mmHg}$ or diastolic BP $>85 \mathrm{mmHg}$ measured by ambulatory BP monitoring from medical records at the start of dialysis, or the use of antihypertensive medications [6]. The presence of CVD were defined as coronary heart disease, congestive heart failure, arrhythmias, cerebrovascular disease, or peripheral vascular disease. Current smoking was defined as at least one cigarette a day, and 
current alcohol consumption defined as $>20$ grams of ethanol a day [15]. eGFR was calculated using the Chronic Kidney Disease Epidemiology Collaboration equation [16].

\section{Outcomes and Follow-Up}

The primary and secondary outcomes were all-cause and CVD mortality, respectively. If the patients died in any hospital, the exact cause of death was available by death certificates, and if the patients died outside a hospital, experts would meet a consensus on the cause of death, with a comprehensive consideration of recent health conditions provided by family members, and the medical history and descriptions from each dialysis center. Patients who died within three months from transferring to hemodialysis to death were considered to receive failure of CAPD therapy and not to be censored. All participants were conducted CAPD schedules in the light of International Standardized Peritoneal Dialysis Guidelines[17], and clinical nephrologists regularly adjusted the CAPD regimens according to patient's health conditions. All patients were followed up until CAPD cessation, death, the end of 8-year duration, or as of June 30,2019. Transferring to hemodialysis with survival time of at least three months, renal transplantation, transferring to other centers, loss of follow-up, or still survival with a follow-up period of 8 years were considered to be censored.

\section{Statistical Analysis}

Variables with missing data before the data analysis were imputed using the missForest method, coping with different types of variables [18]. Incidence was calculated as number of events divided by total valid observational time at risk, scaled to episodes per 1000 years. Variables are presented as mean \pm standard deviation (SD) or median (interquartile range, IQR) or number (\%). Patients were divided into four groups: control group (those without either HTN or pre-existing CVD), HTN group, CVD group, and HTN plus CVD group. Baseline variables were compared by the One-Way ANOVA or Kruskal-Wallis tests according to variable distribution (normality tested with Shapiro-Wilk test) for quantitative variables, and the chisquare test when appropriate for categorical variables among the groups. Multiple Logistic regression was conducted to evaluate the association between baseline variables and the co-existence of HTN and pre-existing CVD at baseline. The following factors were included in multiple Logistic regression: age, sex, body mass index, systolic BP, diastolic BP, current smoking, current alcohol consumption, 24-hour urine volume, diabetes mellitus, hyperlipidemia, hemoglobin, serum albumin, serum uric acid, eGFR, cholesterol, triglyceride, high density lipoprotein, low density lipoprotein, and hs-CRP. Medications were not included in the multiple Logistic regression because of the following reason: medications were usually used in those who had developed comorbidities, but not those comorbidity-free patients. If we enrolled medications into the multiple Logistic regression, the findings must showed that patients with the usage of medications are at higher risk for the presence of HTN plus and pre-existing CVD compared to those without the usage of medications, which was contradicted with clinical knowledge. Therefore, we did not included the usage of medications in multiple Logistic regression.

We used Kaplan-Meier curves to investigate the difference of cumulative mortality among four groups over the observational period. To analyze the association between comorbidities and mortality, we 
constructed four Cox proportional hazards regression models adjusted for the following factors: Model 1 , unadjusted; model 2, model 1 plus age, sex, body mass index, systolic BP, diastolic BP, current smoking, current alcohol consumption, 24-hour urine volume, diabetes mellitus, and hyperlipidemia; model 3, model 2 plus medications; model 4, model 3 plus hemoglobin, serum albumin, serum uric acid, eGFR, cholesterol, triglyceride, high density lipoprotein, low density lipoprotein, and hs-CRP. In addition, the association between comorbidities and mortality were also analyzed among subgroups of men, women, diabetes mellitus, non-diabetes mellitus, hyperlipidemia, and non-hyperlipidemia. The interaction between HTN and CVD on all-cause and CVD mortality was examined by performing a formal test of interaction.

\section{Sensitivity Analysis}

First, for all-cause mortality, hemodialysis or renal transplants were considered competing risks. When using hemodialysis or renal transplants as competing risks, we evaluated comorbidities and all-cause mortality using the Fine and Gray competing risk models, with adjusting for variables in the four Cox proportional hazards regression models. Similarly, for CVD mortality, non-CVD mortality, hemodialysis or renal transplants were considered competing risks. Second, for those adult patients with a short-term period of follow-up, the interesting outcomes may not be completely observed, with under-reporting of the incidence of mortality. For fully observing outcomes, we further analyzed the effect of comorbidities at the start of dialysis on mortality in those adult patients with at least 24-month period of follow-up.

The results of the Cox proportional hazards models and the Fine and Gray models were presented as the hazard ratio (HR) and the $95 \%$ confidence interval $(\mathrm{Cl})$. Statistical analyses were conducted by GraphPad Software 8.0 (GraphPad Prism Software Inc., San Diego, California) and the R package 3.6.0 (https://www.r-project.org/). The level of significance was set as 0.05 for all analyses.

\section{Results}

\section{Patient Characteristics and comorbidities}

All 3073 incident CAPD patients from five dialysis centers were included in the present study. All variables with less than $5 \%$ missing data were imputed before the data analysis, and there was no missing data for outcomes. Of 3073 with a median age of 49.0 (IQR 39.0-61.0), 1780 (57.9\%) were men, 1987 (64.6\%) had HTN, 431 (14.1\%) had pre-existing CVD, and 567 (18.4\%) had diabetes mellitus. All patients were divided into four group: HTN plus CVD group ( $n=370,12.0 \%)$, CVD group ( $n=60,2.0 \%), \operatorname{HTN}(52.6 \%)$ group, and the control group ( $n=1027,33.4 \%$, Table 1). Compared to the control group, HTN plus CVD group tended to be elderly, with higher body mass index, systolic BP, hemoglobin, and cholesterol, but lower diastolic BP, as well as more likely to be current smoking, diabetes mellitus, hyperlipidemia, taking calcium channel blockers, beta blockers, diuretics, ACEI/ARBs, aspirin, and statins.

\section{Co-existence of HTN plus preexisting CVD at baseline and Related factors}


We analyzed the factors associated with the co-existence of HTN and CVD at baseline using the multiple Logistic regression in the study population (Table 2). When adjusting for body mass index, diastolic BP, 24-hour urine volume, current alcohol consumption, serum albumin, serum uric acid, eGFR, cholesterol, triglyceride, high density lipoprotein, low density lipoprotein, and hs-CRP, we found that age, sex, systolic $\mathrm{BP}$, current smoking, diabetes mellitus, hyperlipidemia, and hemoglobin were independently associated with the co-existence of HTN and pre-existing CVD.

\section{Observational Period and Mortality}

The median observational period was 33.7 (IQR 15.7-60.9) months. During this period, $571(18.6 \%, 95 \% \mathrm{Cl}$ 17.1 to $20.0 \%)$ of 3073 patients died, with 286 (9.3\%, 95\% Cl 8.2\% to $10.4 \%)$ CVD mortality, 375 (12.2\%, $95 \% \mathrm{Cl} 11.2 \%$ to $13.4 \%)$ transferring to hemodialysis, $159(5.2 \%, 95 \% \mathrm{Cl} 4.5 \%$ to $6.0 \%)$ receiving renal transplants, $26(0.8 \%, 95 \% \mathrm{Cl} 0.6 \%$ to $1.2 \%)$ transferring to other dialysis centers, and $106(3.4 \%, 95 \% \mathrm{Cl}$ $2.8 \%$ to $4.1 \%)$ loss of follow-up. The number of all-cause mortality was $143(38.6 \%, 95 \% \mathrm{Cl} 33.7 \%$ to $44.2 \%$ ), 15 (25.0\%, 95\% Cl 14.6\% to $36.0 \%), 293$ (18.1\%, 95\% Cl $16.2 \%$ to $20.1 \%)$, and $120(11.7 \%, 95 \% \mathrm{Cl}$ $9.7 \%$ to $13.5 \%$ ) in the HTN plus CVD group, CVD group, HTN group, and control group, respectively. The number of CVD mortality was $76(20.5 \%, 95 \% \mathrm{Cl} 16.5 \%$ to $25.0 \%), 7(11.7 \%, 95 \% \mathrm{Cl} 4.8 \%$ to $21.1 \%), 147$ $(9.1 \%, 95 \% \mathrm{Cl} 7.7 \%$ to $10.6 \%)$, and $56(5.5 \%, 95 \% \mathrm{Cl} 4.2 \%$ to $6.8 \%)$ in the HTN plus CVD group, CVD group, HTN group, and control group, respectively.

The incidence of all-cause mortality was $55.7 / 1000$ patient-years in the study population, with $27.9 / 1000$ patient-years of CVD mortality incidence (Table 3). The incidence of all-cause mortality was 131.0, 74.4, 56.1, and 32.2/1000 patient-years, and CVD mortality incidence was 69.6, 34.7, 28.2, and 15.0/1000 patient-years among the HTN plus CVD group, CVD group, HTN group, and control group, respectively.

\section{Comorbidities and Mortality}

Survival analysis found that the HTN plus CVD group had poorer cumulative survival $(P<0.001)$ and CVD mortality-free survival $(P=0.006)$ compared to the control group (Figure 1). The association between comorbidities and mortality was evaluated by the different Cox proportional hazards regression models (Table 4). When comparing to the control group, the HTN plus CVD group, CVD group and HTN group had $3.98(95 \% \mathrm{Cl} 3.07$ to 5.17$), 2.18$ ( $95 \% \mathrm{Cl} 1.27$ to 3.74$)$, and 1.83 ( $95 \% \mathrm{Cl} 1.47$ to 2.28$)$-time risk of all-cause morality in the model 4, respectively. The HTN plus CVD group, CVD group and HTN group had 4.68 ( $95 \% \mathrm{Cl} 3.27$ to 6.69$), 2.11(95 \% \mathrm{Cl} 0.96$ to 4.63$)$, and 1.87 (95\% $\mathrm{Cl} 1.37$ to 2.54 )-time risk for CVD mortality compared to the control group in the model 4, respectively. Similar trends of the association between comorbidities and mortality were observed among subgroups of men, women, diabetes mellitus, nondiabetes mellitus, hyperlipidemia, and non-hyperlipidemia (Figure 2). There was no significant interaction between HTN and CVD on all-cause and CVD mortality $(\beta=0.010, P=0.973 ; \beta=0.058, P=0.892)$ in the study population.

\section{Sensitivity Analysis}


When performing competing risk analyses with hemodialysis or renal transplants as the competing risk factors, the HTN plus CVD group, CVD group and HTN group had 3.00 (95\% $\mathrm{Cl} 2.19$ to 4.11$), 2.03$ (95\% Cl 1.11 to 3.73$)$, and $1.37(95 \% \mathrm{Cl} 1.07$ to 1.75$)$-time risk of all-cause morality compared to the control group, respectively, in the Fine and Gray model 4. Similarly, when using non-CVD mortality, hemodialysis or renal transplants as the competing risk factors, compared to the control group, the HTN plus CVD group, CVD group and HTN group had 3.17 ( $95 \% \mathrm{Cl} 2.03$ to 4.97$), 2.01$ ( $95 \% \mathrm{Cl} 0.83$ to 4.89$)$, and $1.54(95 \% \mathrm{Cl} 1.08$ to 2.18)-time risk of all-cause morality, respectively, in the Fine and Gray model 4.

A total of $42(1.4 \%)$ patients aged $<18$ years at the start dialysis were excluded, with 6 deaths at the end study. By the end of study, 810 (26.3\%) adult patients were follow up less than 24 months, and 282 (9.2\%) adult patients survived for less than 24 months. The remaining $1939(63.1 \%)$ adult patients were follow up for at least 24 months, with $283(14.6 \%, 95 \% \mathrm{Cl} 12.9 \%$ to $16.1 \%)$ of all-cause mortality and 135 (7.0\%, 95\% Cl $5.8 \%$ to $8.1 \%$ ) of CVD mortality. We found that when comparing to the control group, the HTN plus CVD group, CVD group, and HTN group had 3.53 (95\% Cl 2.32 to 4.84), 1.73 (95\% Cl 1.01 to 2.97), and 1.46 (95\% $\mathrm{Cl} 1.17$ to 1.81)-time risk of all-cause mortality, and 4.59 (95\% $\mathrm{Cl} 2.70$ to 7.79 ), 1.93 (95\% $\mathrm{Cl} 0.88$ to 4.28 ), and 1.58 (95\% $\mathrm{Cl} 1.15$ to 2.16 )-time risk of CVD mortality in the Cox regression model 4, respectively, among adult those with at least 24-month follow-up period.

\section{Discussion}

In our multi-center study of 3073 incident Chinese CAPD patients, the co-existence of HTN and preexisting CVD at the start of dialysis were more strongly associated with all-cause and CVD mortality compared to either HTN or pre-existing CVD alone. Pre-existing CVD was also more strongly associated with all-cause and CVD mortality than hypertension. Similar trends were observed in the competing risk analysis, subgroups of men, women, diabetes mellitus, non-diabetes mellitus, hyperlipidemia, and nonhyperlipidemia patients, as well as those with at least 24-month period of follow up.

HTN is high prevalent and plays a significant role in the mortality of dialysis patients [19]. Previous observational studies over the past decade have confirmed the "U-shaped" or "reverse J-shaped" relationship between BP and mortality of dialysis patients [20-23]. On the contrary, a direct linear association between systolic BP outside the unit and all-cause mortality was observed (HR 1.26 for each $10 \mathrm{mmHg}$ higher systolic $\mathrm{BP} ; 95 \% \mathrm{Cl} 1.14$ to1.40) [24]. However, the relationship between HTN, as a comorbidity, and mortality of dialysis patients has received little attention. Meanwhile, the prevalence of CVD was $64.5 \%$ among patients aged $>65$ years with chronic kidney disease, compared to $32.4 \%$ among those without CKD. Thus, dialysis patients may have a higher prevalence of pre-existing CVD, when receiving RRT, and have poorer survival. A study of 107,922 dialysis patients from the Unite States evaluated the association between the dialysis modality and mortality, with $26.0 \%$ of coronary artery disease of new ESRD patients [12]. The HR of death was significantly greater for patients with coronary artery disease compared with those without these conditions at ESRD onset CAD (HR 1.11,95\% $\mathrm{Cl} 1.08$ to 1.14). We previously conducted a study of 1068 Chinese CAPD patients, with $30.8 \%$ of pre-existing CVD patients. This reported that $7.0 \%$ of prior stroke CAPD patients $(n=75)$ had 1.82 -time risk of all-cause 
mortality than those without this condition. However, to best of our knowledge, there has been no study focusing on the association between the co-existence of HTN and pre-existing CVD in dialysis patients. In the present study, we first reported that the co-existence of HTN and pre-existing CVD were more strongly associated with all-cause and CVD mortality compared to either HTN or pre-existing CVD alone in CAPD patients, with similar findings from the competing risk analysis. The $64.6 \%$ of prevalence of HTN in our study was slightly lower that those in these previous studies, in which the prevalence of HTN was $65.7 \%$ and $73.8 \%$ in Chinese CAPD patients $[4,5]$. The $14.1 \%$ of prevalence of pre-existing CVD was mildly higher than $10.5 \%$ pre-existing CVD in one single study with 740 incident Chinese CAPD patients[5], but significantly lower than $30.3 \%$ in another single study with 985 incident Chinese CAPD patients [4]. In the study population, we found that when using those without either HTN or pre-existing CVD as a reference, patients with only pre-existing CVD had also more strongly associated with all-cause mortality (HR 2.18 vs. HR 1.83) and CVD mortality (HR 2.11 vs. HR 1.87) than those with only HTN. Among patients with at least 24-month survival time, patients with only pre-existing CVD had also more strongly associated with all-cause mortality (HR 1.73 vs. HR 1.46) and CVD mortality (HR 1.93 vs. HR 1.1.58) than those with only HTN, when comparing to those without either HTN or pre-existing CVD alone. Notably, the 2.18 of HR of pre-existing CVD for all-cause mortality was significantly higher than 1.11 of HR in the above-mentioned study with 2-year follow-up period [12], but slightly higher than 1.82 of HR in our previous study [13]. The disparities of these findings may be due to (1) different ethnicity; (2) different sample size; (3) different follow-up duration.

In the present study, baseline variables were unmatched among groups. Compared to patients with only HTN, those with HTN and pre-existing CVD were more likely to be elderly age, higher percentiles of current smoking, diabetes mellitus, and hyperlipidemia, higher levels of systolic BP, hemoglobin, and cholesterol. Elderly age, current smoking, and diabetes mellitus had an adverse effect on the prognosis of dialysis patients [25-28]. Patients with HTN and pre-existing CVD had higher percentiles of diabetes mellitus and hyperlipidemia than patients with only HTN, which contributed to higher percentiles of taking medications. Therefore, patients with HTN and pre-existing CVD had higher percentiles of taking calcium channel blockers, beta blockers, diuretics, ACEI/ARBs, aspirin, and statins. In summary, we should cautiously interpret our findings due to the significant difference of baseline variables among the study population.

Strengths of this study included a large sample size, population from five dialysis centers, and a detailed evaluation and adjustment for all-cause and CVD risk factors of real-world data. Several limitations should be considered. First, this was a retrospective study with potential unaccounted confounding factors and the selection biases. Although after adjusting for baseline variables, we did not draw conclusions about potential causal relationship between comorbidities and mortality. Nonetheless, changes of HRs among the model 2, 3, and 4 were less than $10 \%$, suggesting three models we built were stable and reliable for predicting outcomes [29]. Second, one challenge was the definition of HTN. The optimal method to diagnose HTN in peritoneal dialysis patients is an area of controversy [30]. A recent study reported that similarly to the general populations, ambulatory BP monitoring is the gold standard method in management of HTN in peritoneal dialysis patients [6]. In our study, diagnosis of HTN mainly 
based on the BP value measured by the ambulatory BP monitoring from their medical records, or the use of antihypertensive medications. Nonetheless, we did not analyzed the effect of changes of systolic BP and diastolic BP on the mortality in CAPD patients. Lastly, all eligible patients were from China, suggesting our findings may lack generalization to other ethnic populations.

In conclusion, the co-existence of HTN and pre-existing CVD at the start of CAPD had a more harmful effect on the subsequent risk of all-cause and CVD mortality than either HTN or pre-existing CVD alone, and pre-existing CVD was also more strongly associated with risk of all-cause and CVD mortality than HTN among CAPD patients. Our findings suggested that a combined assessment of HTN and pre-existing CVD compared with separate assessment of the two comorbidities further improved risk stratification of CAPD patients at risk of mortality.

\section{List Of Abbreviations}

CAPD, continuous ambulatory peritoneal dialysis; HTN, hypertension; CVD, cardiovascular disease; BP, blood pressure; ACEI/ARB, beta blockers, angiotensin II receptor blockers/angiotensin-converting enzyme inhibitors; eGFR, estimated glomerular filtration rate; hs-CRP, high-sensitivity C-reactive protein; OR, odds ratio; $\mathrm{HR}$, hazards ratio; $\mathrm{Cl}$, confidence index.

\section{Declarations}

\section{Ethics approval and consent to participate}

The study was consistent with the ethical principles of the Declaration of Helsinki and was approved by the Human Ethics Committee of the Second Affiliated Hospital of Guangzhou Medical University, Zhujiang Hospital of Southern Medical University, Jiujiang No. 1 People's Hospital, Affiliated Sixth People's Hospital, Shanghai Jiao Tong University, The First Affiliated Hospital of Zhengzhou University, and the First Affiliated Hospital of Nanchang University. Written informed consent was obtained from all participants.

\section{Consent for publication}

All authors have approved the submitted version. All authors have agreed both to be personally accountable for the author's own contributions and to ensure that questions related to the accuracy or integrity of any part of the work, even ones in which the author was not personally involved, are appropriately investigated, resolved, and the resolution documented in the literature.

\section{Availability of data and materials}


The datasets used and/or analyzed during the current study are available from the corresponding author on reasonable request.

\section{Competing interests}

The authors declare that they have no competing interests.

\section{Funding}

No.

\section{Authors' contributions}

$X Y$ W, contributions to the conception, and drafted the work; XR F, the acquisition of data; FF P, the acquisition of data; $Y Q W$, the acquisition of data; NS W, contributions to the conception and design of the work; Q, Z, analysis and interpretation of data; $X J \mathrm{Z}$, contributions to the conception and design of the work; XF W, contributions to the conception, design of the work, and revised it. All authors have read and approved the manuscript.

\section{Acknowledgements}

We express our gratitude to all patients who participated in the study.

\section{References}

1. Kendrick J, Teitelbaum I. Strategies for improving long-term survival in peritoneal dialysis patients. Clin J Am Soc Nephrol. 2010;5(6):1123-31.

2. Hall YN, Chertow GM: End stage renal disease. BMJ Clin Evid 2007, 2007.

3. Jha V, Garcia-Garcia G, Iseki K, Li Z, Naicker S, Plattner B, Saran R, Wang AY, Yang CW. Chronic kidney disease: global dimension and perspectives. Lancet. 2013;382(9888):260-72.

4. Xia X, He F, Wu X, Peng F, Huang F, Yu X. Relationship between serum uric acid and all-cause and cardiovascular mortality in patients treated with peritoneal dialysis. Am J Kidney Dis. 2014;64(2):257-64.

5. Zhan X, Yang M, Chen Y, Yan C, Wang Y, Zhao Q, Chen Q, Zhang L. Relationship between serum bilirubin levels and mortality in patients on peritoneal dialysis. Ren Fail. 2019;41(1):532-9. 
6. Agarwal R, Flynn J, Pogue V, Rahman M, Reisin E, Weir MR. Assessment and management of hypertension in patients on dialysis. J Am Soc Nephrol. 2014;25(8):1630-46.

7. Fang W, Yang X, Bargman JM, Oreopoulos DG. Association between pulse pressure and mortality in patients undergoing peritoneal dialysis. Perit Dial Int. 2009;29(2):163-70.

8. Udayaraj UP, Steenkamp R, Caskey FJ, Rogers C, Nitsch D, Ansell D, Tomson CR. Blood pressure and mortality risk on peritoneal dialysis. Am J Kidney Dis. 2009;53(1):70-8.

9. Briasoulis A, Bakris GL. The OSCAR for cardiovascular disease prevention in chronic kidney disease goes to blood pressure control. Kidney Int. 2013;83(1):20-2.

10. Sarnak MJ, Levey AS, Schoolwerth AC, Coresh J, Culleton B, Hamm LL, McCullough PA, Kasiske BL, Kelepouris E, Klag MJ, et al. Kidney disease as a risk factor for development of cardiovascular disease: a statement from the American Heart Association Councils on Kidney in Cardiovascular Disease, High Blood Pressure Research, Clinical Cardiology, and Epidemiology and Prevention. Circulation. 2003;108(17):2154-69.

11. US Renal Data System 2018 Annual Data Report:

Saran R, Robinson B, Abbott KC, Agodoa LYC, Bragg-Gresham J, Balkrishnan R, Bhave N, Dietrich X, Ding Z, Eggers PW, et al: US Renal Data System 2018 Annual Data Report: Epidemiology of Kidney Disease in the United States. Am J Kidney Dis 2019, 73(3 Suppl 1):A7-8.

12. Ganesh SK, Hulbert-Shearon T, Port FK, Eagle K, Stack AG. Mortality differences by dialysis modality among incident ESRD patients with and without coronary artery disease. J Am Soc Nephrol. 2003;14(2):415-24.

13. Wu X, Yang X, Liu X, Yi C, Guo Q, Feng X, Mao H, Huang F, Yu X. Patient Survival and Technique Failure in Continuous Ambulatory Peritoneal Dialysis Patients with Prior Stroke. Perit Dial Int. 2016;36(3):308-14.

14. Ma L, Zhao S. Risk factors for mortality in patients undergoing hemodialysis: A systematic review and meta-analysis. Int J Cardiol. 2017;238:151-8.

15. Tu W, Wu J, Jian G, Lori J, Tang Y, Cheng H, Wu X, Wang N. Asymptomatic hyperuricemia and incident stroke in elderly Chinese patients without comorbidities. Eur J Clin Nutr. 2019;73(10):1392402.

16. Zhang L, Wang F, Wang L, Wang W, Liu B, Liu J, Chen M, He Q, Liao Y, Yu X, et al. Prevalence of chronic kidney disease in China: a cross-sectional survey. Lancet. 2012;379(9818):815-22.

17. Woodrow G, Fan SL, Reid C, Denning J, Pyrah AN. Renal Association Clinical Practice Guideline on peritoneal dialysis in adults and children. BMC Nephrol. 2017;18(1):333.

18. Stekhoven DJ, Buhlmann P. MissForest-non-parametric missing value imputation for mixed-type data. Bioinformatics. 2012;28(1):112-8.

19. Agarwal R, Nissenson AR, Batlle D, Coyne DW, Trout JR, Warnock DG. Prevalence, treatment, and control of hypertension in chronic hemodialysis patients in the United States. Am J Med. 2003;115(4):291-7. 
20. Port FK, Hulbert-Shearon TE, Wolfe RA, Bloembergen WE, Golper TA, Agodoa LY, Young EW. Predialysis blood pressure and mortality risk in a national sample of maintenance hemodialysis patients. Am J Kidney Dis. 1999;33(3):507-17.

21. Zager PG, Nikolic J, Brown RH, Campbell MA, Hunt WC, Peterson D, Van Stone J, Levey A, Meyer KB, Klag MJ, et al. "U" curve association of blood pressure and mortality in hemodialysis patients. Medical Directors of Dialysis Clinic, Inc. Kidney Int. 1998;54(2):561-9.

22. Tozawa M, Iseki K, Iseki $C$, Takishita S. Pulse pressure and risk of total mortality and cardiovascular events in patients on chronic hemodialysis. Kidney Int. 2002;61(2):717-26.

23. Li Z, Lacson E Jr, Lowrie EG, Ofsthun NJ, Kuhlmann MK, Lazarus JM, Levin NW. The epidemiology of systolic blood pressure and death risk in hemodialysis patients. Am J Kidney Dis. 2006;48(4):60615.

24. Bansal N, McCulloch CE, Rahman M, Kusek JW, Anderson AH, Xie D, Townsend RR, Lora CM, Wright $\mathrm{J}, \mathrm{Go}$ AS, et al. Blood pressure and risk of all-cause mortality in advanced chronic kidney disease and hemodialysis: the chronic renal insufficiency cohort study. Hypertension. 2015;65(1):93-100.

25. Canaud B, Tong L, Tentori F, Akiba T, Karaboyas A, Gillespie B, Akizawa T, Pisoni RL, Bommer J, Port FK. Clinical practices and outcomes in elderly hemodialysis patients: results from the Dialysis Outcomes and Practice Patterns Study (DOPPS). Clin J Am Soc Nephrol. 2011;6(7):1651-62.

26. Byrne $C$, Vernon P, Cohen JJ. Effect of age and diagnosis on survival of older patients beginning chronic dialysis. JAMA. 1994;271(1):34-6.

27. Formanek P, Salisbury-Afshar E, Afshar M. Helping Patients With ESRD and Earlier Stages of CKD to Quit Smoking. Am J Kidney Dis. 2018;72(2):255-66.

28. Rhee CM, Leung AM, Kovesdy CP, Lynch KE, Brent GA, Kalantar-Zadeh K. Updates on the management of diabetes in dialysis patients. Semin Dial. 2014;27(2):135-45.

29. Kernan WN, Viscoli CM, Brass LM, Broderick JP, Brott T, Feldmann E, Morgenstern LB, Wilterdink JL, Horwitz RI. Phenylpropanolamine and the risk of hemorrhagic stroke. N Engl J Med. 2000;343(25):1826-32.

30. Vaios V, Georgianos PI, Liakopoulos V, Agarwal R. Assessment and Management of Hypertension among Patients on Peritoneal Dialysis. Clin J Am Soc Nephrol. 2019;14(2):297-305.

\section{Tables}

Table 1. The demographic characteristics, medications, and laboratory parameters at the start of dialysis therapy among four groups. 


\begin{tabular}{|c|c|c|c|c|c|c|}
\hline & $\begin{array}{c}\text { Study } \\
\text { population }\end{array}$ & $\begin{array}{l}\text { Control } \\
\text { group }\end{array}$ & HTN group & CVD group & $\begin{array}{l}\text { HTN plus } \\
\text { CVD group }\end{array}$ & $\begin{array}{c}\mathrm{P}- \\
\text { value }\end{array}$ \\
\hline ber & 3073 & 1027 & 1616 & 60 & 370 & \\
\hline years & $\begin{array}{c}49.0(39.0- \\
61.0)\end{array}$ & $\begin{array}{c}45.0(34.0- \\
56.0)\end{array}$ & $\begin{array}{c}49.0(39.0- \\
60.0)\end{array}$ & $\begin{array}{c}54.0(44.0- \\
64.0)\end{array}$ & $\begin{array}{c}62.0(52.0- \\
70.0)\end{array}$ & $<0.001$ \\
\hline stratification & & & & & & $<0.001$ \\
\hline years & $\begin{array}{c}876 \\
(28.5 \%)\end{array}$ & $\begin{array}{c}403 \\
(39.2 \%)\end{array}$ & $\begin{array}{c}436 \\
(27.0 \%)\end{array}$ & $12(20.0 \%)$ & 25 (6.8\%) & \\
\hline 50 years & $\begin{array}{c}741 \\
(24.1 \%)\end{array}$ & $\begin{array}{c}253 \\
(24.8 \%)\end{array}$ & $\begin{array}{c}419 \\
(25.9 \%)\end{array}$ & $15(25.0 \%)$ & 54 (14.6\%) & \\
\hline 60 years & $\begin{array}{c}665 \\
(21.7 \%)\end{array}$ & $\begin{array}{c}202 \\
(19.8 \%)\end{array}$ & $\begin{array}{c}364 \\
(22.5 \%)\end{array}$ & 12 (20.0\%) & $87(23.5 \%)$ & \\
\hline 70 years & $\begin{array}{c}539 \\
(17.6 \%)\end{array}$ & $\begin{array}{c}125 \\
(12.2 \%)\end{array}$ & $\begin{array}{c}284 \\
(17.6 \%)\end{array}$ & $14(23.3 \%)$ & $\begin{array}{c}116 \\
(31.4 \%)\end{array}$ & \\
\hline years & $252(8.2 \%)$ & $43(4.2 \%)$ & $114(7.1 \%)$ & $7(11.7 \%)$ & 88 (23.8\%) & \\
\hline$\%$ & $\begin{array}{c}1780 \\
(57.9 \%)\end{array}$ & $\begin{array}{c}568 \\
(55.3 \%)\end{array}$ & $\begin{array}{c}957 \\
(59.2 \%)\end{array}$ & $30(50.0 \%)$ & $\begin{array}{c}225 \\
(60.8 \%)\end{array}$ & 0.080 \\
\hline
\end{tabular}


2

lic BP, $\begin{array}{llllll}149.8 \pm 25.7 & 143.1 \pm 26.1 & 153.2 \pm 24.5 & 135.9 \pm 26.8 & 154.6 \pm 25.6 & <0.001\end{array}$ [g :olic BP, $\quad 87.5 \pm 15.8 \quad 85.3 \pm 15.8 \quad 89.6 \pm 15.6 \quad 84.4 \pm 15.7 \quad 84.5 \pm 15.3<0.001$ [g 800(500- $\quad 800(440-\quad 800(500-\quad 900(400-\quad 800(450-\quad 0.861$ urine volume, ent smoking, 310 $75(7.3 \%)$ 184 $2(3.3 \%)$ $49(13.2 \%)<0.001$ $(10.1 \%)$ $(11.4 \%)$

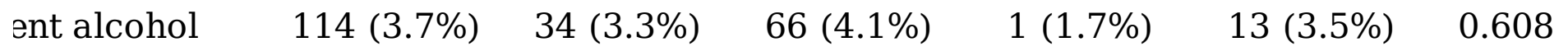
umption, (\%) 


$\begin{array}{lcccccc}\text { etes mellitus, } & 567 & 67(6.5 \%) & 318 & 7(11.7 \%) & 175 & <0.001 \\ & (18.4 \%) & & (19.7 \%) & & (47.3 \%) & \\ \text { rlipidemia } & 567 & 140 & 282 & 20(33.3 \%) & 125 & <0.001 \\ & (18.4 \%) & (13.6 \%) & (17.5 \%) & & (33.8 \%) & \\ \text { um channel } & 2271 & 608 & 1311 & 36(60.0 \%) & 315 & <0.001 \\ \text { ters (\%) } & (73.9 \%) & (59.2 \%) & (81.1 \%) & & (85.1 \%) & \\ \text { blockers (\%) } & 1255 & 362 & 695 & 17(28.3 \%) & 181 & <0.001 \\ & (40.8 \%) & (35.2 \%) & (43.0 \%) & & (48.9 \%) & \end{array}$

tics (\%) $205(6.7 \%) \quad 32(3.1 \%) \quad 123(7.6 \%)$

/ARBs (\%)

$\begin{array}{ccc}1042 & 293 & 556 \\ (33.9 \%) & (28.5 \%) & (34.4 \%)\end{array}$

in $(\%)$

$247(8.0 \%) \quad 30(2.9 \%) \quad 128(7.9 \%)$

ns (\%)

439

$73(7.1 \%)$

241

$1(1.7 \%)$

$49(13.2 \%)<0.001$

(14.3\%)

(14.9\%)

$19(31.7 \%)$

173

$<0.001$

(46.8\%)

Jglobin, g/dL

m albumin,

$9.3 \pm 2.8$

$9.2 \pm 2.9$

$9.1 \pm 2.8$

$9.1 \pm 2.2$

$86(23.2 \%)<0.001$

$3.5 \pm 0.6$

$3.5 \pm 0.6$

$3.5 \pm 0.5$

$3.4 \pm 0.7$

113

$<0.001$

(30.5\%)

m uric acid,

$6.9 \pm 2.3 \quad 7.0 \pm 2.3$

$6.9 \pm 2.4$

$6.4 \pm 2.2$

$9.8 \pm 2.9<0.001$ L

?,

$6.4(4.7-\quad 6.6(4.7-\quad 6.4(4.7-$

$6.1(4.6-$

$3.5 \pm 0.6$

0.750

$\operatorname{nin} / 1.73 \mathrm{~m}^{2}$

əsterol,

L

yceride,

L

density

8.3)

8.5)

8.2)

8.4)

$6.2(4.7-$

0.415

151 (117- $146(112-\quad 153$ (118

157 (132-

8.2)

$$
\text { 183) }
$$

179)

183)

187)

157 (125-

0.045

$94(57$

92 (62-

95 (53-

149)

156)

85 (32-

187)

153)

$40(31-50) \quad 39(31-51) \quad 40(32-50)$

158)

99 (59-

0.413

153)

rotein, $\mathrm{mg} / \mathrm{dL}$

density

$82(48$

82 (54-

$82(4-116)$

89 (37-

118)

120)

79 (30-

0.861

rotein, $\mathrm{mg} / \mathrm{dL}$

PP, mg/L

117)

4.1 (1.8-

4.4 (1.9-

4.5 (2.1-

4.5 (1.7-

116)

$4.4(1.9-\quad 0.643$ 
Control group: patients without either HTN or pre-existing CVD.

HTN, hypertension; CVD, cardiovascular disease; BP, blood pressure; ACEI/ARB, beta blockers, angiotensin II receptor blockers/angiotensin-converting enzyme inhibitors; eGFR, estimated glomerular filtration rate; hs-CRP, high-sensitivity C-reactive protein.

Table 2. Baseline parameters and both the presence of HTN and pre-existing CVD using the multiple Logistic regression.

\begin{tabular}{lcc}
\hline & OR & 95\%CI \\
\hline Age $\leq 40$ years & Reference & \\
\hline Age $41-50$ years & 2.24 & 1.38 to 3.63 \\
\hline Age 51-60 years & 3.36 & 2.11 to 5.35 \\
\hline Age 61-70 years & 5.68 & 3.58 to 9.02 \\
\hline Age $\geq 71$ years & 12.35 & 7.57 to 20.16 \\
\hline Sex (male as a reference) & 0.78 & 0.60 to 1.00 \\
\hline Systolic BP (per increase $10 \mathrm{mmHg})$ & 1.06 & 1.01 to 1.11 \\
\hline Current smoking (yes/no) & 1.48 & 1.02 to 2.15 \\
\hline Diabetes mellitus (yes/no) & 2.87 & 2.23 to 3.69 \\
\hline Hyperlipidemia (yes/no) & 1.81 & 1.40 to 2.37 \\
\hline Hemoglobin (per increase $1 \mathrm{~g} / \mathrm{dL})$ & 1.04 & 1.00 to 1.09 \\
\hline
\end{tabular}

The following parameters at baseline were in the multiple Logistic regression model: age, sex, body mass index, systolic BP, diastolic BP, current smoking, current alcohol consumption, 24-hour urine volume, diabetes mellitus, hyperlipidemia, hemoglobin, serum albumin, serum uric acid, eGFR, cholesterol, triglyceride, high density lipoprotein, low density lipoprotein, and hs-CRP. 
HTN, hypertension; CVD, cardiovascular disease; BP, blood pressure; eGFR, estimated glomerular filtration rate; hs-CRP, high-sensitivity C-reactive protein; OR, odds ratio; CI, confidence interval.

Table 3. All-cause and CVD mortality incidence.

\begin{tabular}{|c|c|c|c|c|c|c|c|}
\hline & $\begin{array}{l}\text { All-cause } \\
\text { mortality }\end{array}$ & $\begin{array}{c}\text { CVD } \\
\text { mortality }\end{array}$ & $\begin{array}{c}\text { Time at } \\
\text { risk } \\
\text { (years) }\end{array}$ & $\begin{array}{c}\text { All-cause } \\
\text { mortality } \\
\text { incidence } \\
\text { (/1000 patient- } \\
\text { years })\end{array}$ & $\begin{array}{c}95 \% \\
\text { CI }\end{array}$ & $\begin{array}{c}\text { CVD } \\
\text { mortality } \\
\text { incidence } \\
\text { (/1000 } \\
\text { patient-years) }\end{array}$ & $\begin{array}{c}95 \% \\
\text { CI }\end{array}$ \\
\hline \multirow[t]{2}{*}{$\begin{array}{l}\text { Study } \\
\text { population }\end{array}$} & 571 & 286 & 10252.5 & 55.7 & $\begin{array}{l}51.3 \\
\text { to }\end{array}$ & 27.9 & $\begin{array}{c}24.6 \\
\text { to }\end{array}$ \\
\hline & & & & & 59.1 & & 30.9 \\
\hline \multirow[t]{2}{*}{$\begin{array}{l}\text { Control } \\
\text { group }\end{array}$} & 120 & 56 & 3729.5 & 32.2 & $\begin{array}{c}27.0 \\
\text { to }\end{array}$ & 15.0 & $\begin{array}{c}11.0 \\
\text { to }\end{array}$ \\
\hline & & & & & 36.9 & & 18.7 \\
\hline \multirow[t]{2}{*}{$\begin{array}{l}\text { HTN } \\
\text { group }\end{array}$} & 293 & 147 & 5221.9 & 56.1 & $\begin{array}{c}52.0 \\
\text { to }\end{array}$ & 28.2 & $\begin{array}{c}23.5 \\
\text { to }\end{array}$ \\
\hline & & & & & 62.8 & & 32.8 \\
\hline \multirow{3}{*}{$\begin{array}{l}\text { CVD } \\
\text { group }\end{array}$} & 15 & 7 & 201.5 & 74.4 & 46.5 & 34.7 & 9.5 \\
\hline & & & & & to & & to \\
\hline & & & & & 112.9 & & 60.1 \\
\hline HTN plus & 143 & 76 & 1091.6 & 131.0 & 116.9 & 69.6 & 54.9 \\
\hline CVD & & & & & to & & to \\
\hline group & & & & & 151.9 & & 83.7 \\
\hline
\end{tabular}

Incidence was calculated as number of events divided by total valid observational time at risk, scaled to episodes per 1000 years. 
Control group: patients without either HTN or pre-existing CVD.

HTN, hypertension; CVD, cardiovascular disease; CI, confidence interval.

Table 4. Adjusted HRs for mortality among different Cox proportional hazards regression models.

\begin{tabular}{cccccccc}
\multicolumn{2}{c}{ Model 1 } & \multicolumn{2}{c}{ Model 2 } & \multicolumn{2}{c}{ Model 3 } & \multicolumn{2}{c}{ Model 4 } \\
\hline HR & 95\%CI & HR & 95\%CI & HR & 95\%CI & HR & 95\%CI
\end{tabular}

All-cause

mortality

Control group Reference

$\begin{array}{lllllllll}\text { HTN group } & 1.74 & 1.41 \text { to } & 1.78 & 1.43 \text { to } & 1.83 & 1.47 \text { to } & 1.83 & 1.47 \text { to }\end{array}$

$\begin{array}{llll}2.15 & 2.20 & 2.27 & 2.28\end{array}$

$\begin{array}{lllllllll}\text { CVD group } & 2.35 & 1.38 \text { to } & 2.18 & 1.28 \text { to } & 2.12 & 1.24 \text { to } & 2.18 & 1.27 \text { to }\end{array}$

$\begin{array}{llll}4.02 & 3.74 & 3.63 & 3.74\end{array}$



$\begin{array}{lllll}\text { group } & 5.20 & 5.26 & 5.09 & 5.17\end{array}$

CVD mortality

Control group Reference

$\begin{array}{lllllllll}\text { HTN group } & 1.87 & 1.38 \text { to } & 1.93 & 1.42 \text { to } & 1.90 & 1.39 \text { to } & 1.87 & 1.37 \text { to }\end{array}$

\begin{tabular}{lcccccccc} 
& & 2.55 & & 2.63 & & 2.59 & 2.54 \\
\hline CVD group & 2.33 & 1.06 to & 2.28 & 1.04 to & 2.20 & 1.01 to & 2.11 & 0.96 to \\
& & 5.11 & & 5.00 & & 4.83 & 4.63 \\
\hline HTN plus CVD & 4.65 & 3.29 to & 4.91 & 3.47 to & 4.68 & 3.27 to & 4.68 & 3.27 to \\
group & & 6.57 & & 6.95 & & 6.69 & 6.69 \\
\hline
\end{tabular}

Model 1, unadjusted; model 2, model 1 plus age, sex, body mass index, systolic BP, diastolic BP, current smoking, current alcohol consumption, 24-hour urine volume, diabetes mellitus, and hyperlipidemia; model 3, model 2 plus medications; model 4, model 3 plus hemoglobin, 
serum albumin, serum uric acid, eGFR, cholesterol, triglyceride, high density lipoprotein, low density lipoprotein, and hs-CRP.

Control group: participants without either HTN or pre-existing CVD.

HTN, hypertension; CVD, cardiovascular disease; BP, blood pressure; eGFR, estimated glomerular filtration rate; hs-CRP, high-sensitivity C-reactive protein; HR, hazard ratio; CI, confidence interval.

Table 5. Adjusted HRs for mortality among the Fine and Gray competing risk models.

\begin{tabular}{|c|c|c|c|c|c|c|c|c|}
\hline & \multicolumn{2}{|c|}{ Model 1} & \multicolumn{2}{|c|}{ Model 2} & \multicolumn{2}{|c|}{ Model 3} & \multicolumn{2}{|c|}{ Model 4} \\
\hline & HR & $95 \% \mathrm{CI}$ & HR & $95 \% \mathrm{CI}$ & HR & $95 \% \mathrm{CI}$ & HR & $95 \% \mathrm{CI}$ \\
\hline \multicolumn{9}{|l|}{ All-cause } \\
\hline \multicolumn{9}{|l|}{ mortality } \\
\hline Control group & Reference & & & & & & & \\
\hline \multirow[t]{2}{*}{ HTN group } & 1.57 & 1.27 to & 1.35 & 1.06 to & 1.41 & 1.10 to & 1.37 & 1.07 to \\
\hline & & 1.95 & & 1.72 & & 1.80 & & 1.75 \\
\hline \multirow[t]{2}{*}{ CVD group } & 2.34 & 1.31 to & 2.22 & 1.24 to & 2.09 & 1.15 to & 2.03 & 1.11 to \\
\hline & & 4.17 & & 3.97 & & 3.79 & & 3.73 \\
\hline HTN plus CVD & 3.52 & 2.51 to & 2.91 & 2.13 to & 3.00 & 2.19 to & 3.00 & 2.19 to \\
\hline group & & 4.94 & & 3.97 & & 4.12 & & 4.11 \\
\hline \multicolumn{9}{|l|}{ CVD mortality } \\
\hline Control group & Reference & & & & & & & \\
\hline \multirow[t]{2}{*}{ HTN group } & 1.73 & 1.28 to & 1.57 & 1.11 to & 1.60 & 1.13 to & 1.54 & 1.08 to \\
\hline & & 2.33 & & 2.23 & & 2.26 & & 2.18 \\
\hline \multirow[t]{2}{*}{ CVD group } & 2.18 & 1.09 to & 2.07 & 0.86 to & 2.04 & 0.84 to & 2.01 & 0.83 to \\
\hline & & 4.37 & & 4.95 & & 4.98 & & 4.89 \\
\hline HTN plus CVD & 4.40 & 2.55 to & 3.19 & 2.04 to & 3.17 & 2.02 to & 3.17 & 2.03 to \\
\hline group & & 7.58 & & 4.98 & & 4.98 & & 4.97 \\
\hline
\end{tabular}


Model 1, unadjusted; model 2, model 1 plus age, sex, body mass index, systolic BP, diastolic BP, current smoking, current alcohol consumption, 24-hour urine volume, diabetes mellitus, and hyperlipidemia; model 3, model 2 plus medications; model 4, model 3 plus hemoglobin, serum albumin, serum uric acid, eGFR, cholesterol, triglyceride, high density lipoprotein, low density lipoprotein, and hs-CRP.

Control group: participants without either HTN or pre-existing CVD.

HTN, hypertension; CVD, cardiovascular disease; BP, blood pressure; eGFR, estimated glomerular filtration rate; hs-CRP, high-sensitivity C-reactive protein; HR, hazard ratio; CI, confidence interval.

\section{Figures}
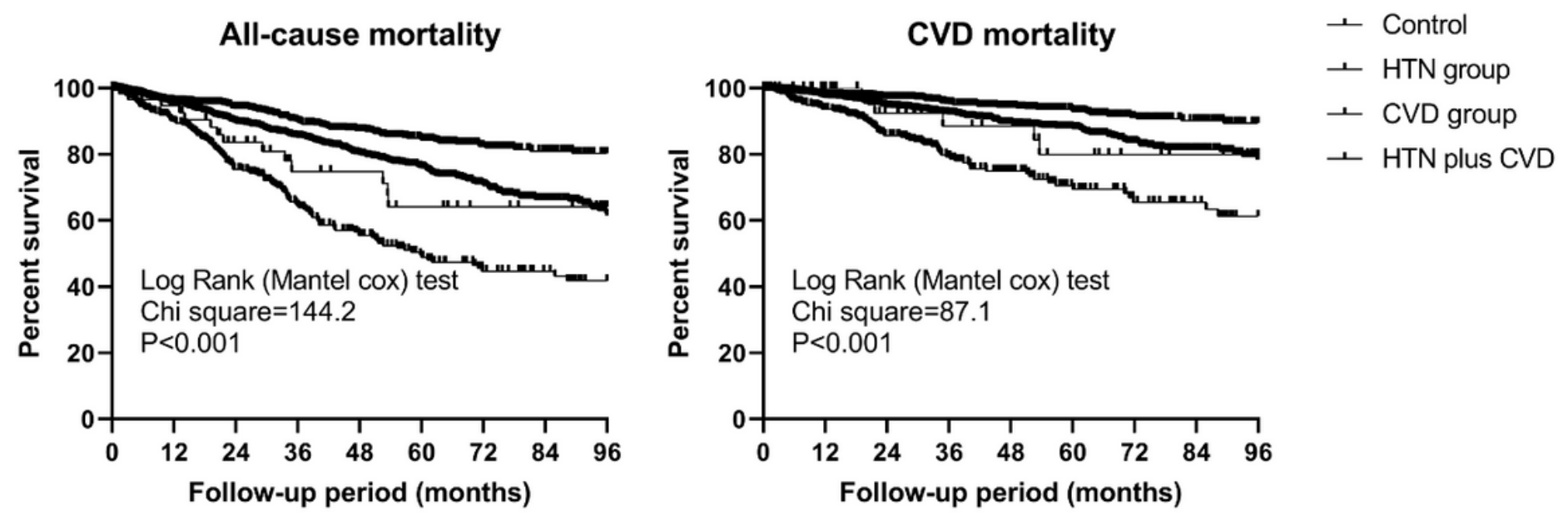

Figure 1

Cumulative survival were lowest in those with both HTN plus pre-existing CVD. Control group: participants without either HTN or pre-existing CVD. HTN, hypertension; CVD, cardiovascular disease. 

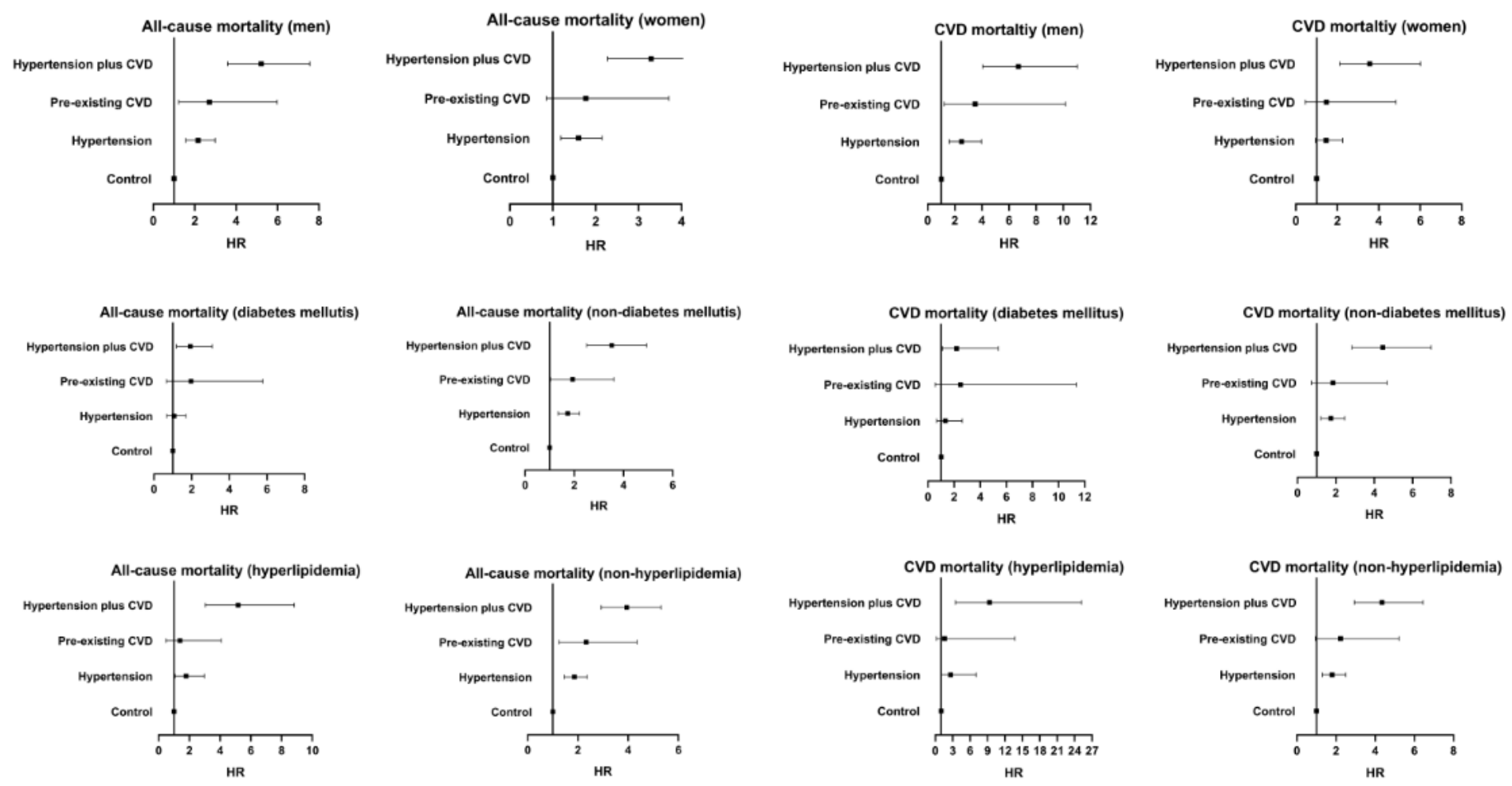

\section{Figure 2}

Adjusted HRs for all-cause and CVD mortality among subgroups. Model 1, unadjusted; model 2, model 1 plus age sex, body mass index, systolic BP, diastolic BP, current smoking, current alcohol consumption, 24-hour urine volume, diabetes mellitus, and hyperlipidemia; model 3, model 2 plus medications; model 4, model 3 plus hemoglobin, serum albumin, serum uric acid, eGFR, cholesterol, triglyceride, high density lipoprotein, low density lipoprotein, and hs-CRP. Control group: participants without either HTN or preexisting CVD. HTN, hypertension; CVD, cardiovascular disease; BP, blood pressure; eGFR, estimated glomerular filtration rate; hs-CRP, high-sensitivity C-reactive protein; $\mathrm{HR}$, hazard ratio; $\mathrm{Cl}$, confidence interval. 